\title{
Hadronic currents and form factors in three-body semileptonic $\tau$ decays
}

\author{
Fabian Krinner ${ }^{1, a}$, Stephan Paul ${ }^{1,2}$ \\ ${ }^{1}$ Max Planck Institut für Physik, 80805 Munich, Germany \\ 2 Physik-Department, Technische Universität München, 85748 Garching, Germany
}

Received: 23 July 2021 / Accepted: 24 November 2021 / Published online: 6 December 2021

(C) The Author(s) 2021

\begin{abstract}
Three-body semileptonic $\tau$-decays offer a path to understand the properties of light hadronic systems and CP symmetry violations through searches for electric dipole moments. In studies of electro-weak physics, the hadronic part of the final states has traditionally been described using the language of form factors. Spectroscopic information, resolved in terms of orbital angular momentum quantumnumbers, is best being derived from an explicit decomposition of the hadronic current in the orbital angular momentum basis. Motivated by the upcoming large data samples from B factories, we present the full description of the hadronic currents decomposed into quantum numbers of the hadronic final state using the isobar picture. We present formulas for orbital angular momenta up to three and apply the rules derived from hadron spectroscopy to formulate the decay chain of hadronic three-body systems of arbitrary mass. We also translate this formalism to the language of form factors and thereby correct insufficiencies found in previous analyses of three-body hadronic final states.
\end{abstract}

\section{Introduction}

The upcoming large data samples from Belle and Belle 2 for $\tau$ decays will be an order of magnitude larger than previously analyzed samples, allowing for analyses of unprecedented detail. The weak decay of the $\tau$ lepton results in leptonic and semi-leptonic final states. The latter can serve as a clean source for hadronic systems produced from the vacuum. The weak current can either be of vector or axial vector nature. Restricting ourselves to light quarks only, we can view the $\mathrm{W}$ Boson to carry strong isospin $I=1$, as it couples to $u \bar{d}$ or $d \bar{u}[1]$. Based on arguments with conserved $G$-parity, odd numbers of pions in the final state can only be produced from the weak axial current, even ones from the weak vector

a e-mail: fkrinner@mpp.mpg.de (corresponding author) current. Contributions through the weak vector current to odd numbers of pions are called second class currents [2,3].

The coupling of the weak axial-vector current to final states with odd numbers of pions can lead to quantum numbers $J^{P}=1^{+}$(axial-vector states) or $J^{P}=0^{-}$(pseudoscalar states) of the hadronic system. Indeed, the decay into a single pseudo-scalar ground-state pion is one of the dominating $\tau$ final states (10.8\% branching fraction [4]). $J^{P}=1^{+}$ axial vectors on the other hand require at least 3 pions in the final state to constitute a first class current not suppressed by $G$ parity. ${ }^{1}$

In this article, we focus on the semileptonic decay of the $\tau$ lepton into three final state pions. Owing to the presence of both vector and axial-vector currents, $\tau$ decays can a priori produce three-pion states with various combinations of quantum numbers $J^{P}$.

Although semileptonic $\tau$ decays have been studied for the last 30 years, the structure of the hadronic current is still not understood sufficiently. Neither has the spectral distribution of the pseudo-scalar current been studied, nor have second class currents been found. In addition, the results on the decay branching for the $\mathrm{a}_{1}(1260)$ shows large deviations from results obtained by direct production in hadronic beam experiments, namely the contribution of the $(\pi \pi)_{S}$-wave in the isobar seems much enhanced in Ref. [5] as compared to other experiments like in Refs. [6,7]. The precise knowledge of the hadronic current, however, is a key requirement for determining the $\tau$ magnetic moment through measurements of the $\tau$ polarization or for searches for an electric dipole moment through spin correlations within the $\tau^{ \pm}$-pair system.

All past analyses of the decay $\tau^{-} \rightarrow 3 \pi \nu_{\tau}$ were restricted to the strong axial-vector component, which is dominantly passing through the $a_{1}(1260)$ resonance. Possible addi-

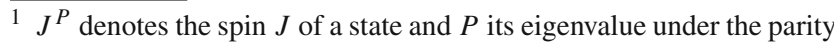
transformation.
} 
tional contributions from pseudo-scalar resonances like the $\pi^{\prime}(1300)$ or from spin-exotic vector resonances like the $\pi_{1}(1600)$, were assumed to have vanishing contributions and were neglected.

Hadronic systems within $\tau$ decays are modeled by a hadronic current consisting of various contributions, called partial waves, that represent various hadronic resonances generating different orbital angular momenta in their decay chains. $^{2}$ Omitting vector and pseudo-scalar partial waves in modelling the $\tau$ decay to three pions, as done previously, led to a very limited set of seven partial waves. Partial waves describing decay chains including orbital angular momenta larger than two units of $\hbar$ were also neglected [5,8].

The presence of partial waves describing $3 \pi$ states other than the $\mathrm{a}_{1}(1260)$ not only allows to study their resonance nature, but also presents the opportunity to study the $\mathrm{a}_{1}$ (1260) itself in the absence of further hadronic interactions. Such interactions are present in alternative production mechanisms, like decays of heavy mesons or the production through strongly interacting hadrons. Possible additional resonances with different $J^{P}$ can serve as phase reference for the $\mathrm{a}_{1}$ (1260), which improves constraints to the fit of the line shape as compared to a mere fit to the intensity distribution alone $[5,11]$.

The detailed study of axial-vectors, vectors, and pseudoscalars in $\tau$-decays requires the derivation of previously omitted contributions to the hadronic current. We will construct these contributions for partial waves with appearing angular momenta of up to three units of $\hbar$ in their decay chain. In Sect. 2, we introduce the general structure of the amplitude and in Sect. 3 we lay out the basic components to formulate hadronic currents. We give the explicit expressions in Sects. 4, 5, and 6. In Sect. 7 we discuss the scope and limitations of our model, relate our findings to the more common language of form factors in Sect. 8, and compare to previous work in Sect. 9. In Sect. 10, we will summarize and discuss the impact of the uncertainty on the hadronic current for the search of a tau lepton electric dipole moment.

\section{The hadronic current}

The amplitude of the semileptonic decay of a $\tau$ lepton into a neutrino and any hadronic final state is governed by the weak interaction and takes the form:

$\mathcal{M} \propto \bar{u}_{v} \gamma_{\mu}\left(1-\gamma^{5}\right) u_{\tau} J_{\text {had }}^{\mu}$

where the left part describes the leptonic current, given by the corresponding Dirac spinors $u_{\tau}$ and $u_{v}$ and the Dirac matri-

2 Note, that we use the term "partial wave analysis" as developed in Ref. [9], rather than the more commonly known definition given in Ref. [10]. ces $\gamma$. The hadronic current $J_{\text {had }}^{\mu}$ is given by the final-state kinematics. The energy released in the decay can be shared among the neutrino and the hadronic final state resulting in a continuous spectrum of the invariant mass of the latter. Since the hadronic current is governed by the strong interaction, there is no ab-initio calculation, but it is usually modeled as a sum of various contributions, which we will call partial waves from hereon:

$J_{\text {had }}^{\mu}=\sum_{w \in \text { waves }} \mathcal{C}_{w} j_{w}^{\mu}$

The sum extends over a set of partial waves $w$ describing the kinematics of the final-state particles. The complex-valued coefficients $\mathcal{C}_{w}$ encode the relative strengths and phases of the individual partial waves and are not known a priori but must be extracted from data via a partial-wave analysis. Even though this approach is applicable to all multi-body hadronic final-states, here we will focus on three-particle final-states, particularly on $\tau^{-} \rightarrow \nu_{\tau}+\pi^{+} \pi^{-} \pi^{-}$.

A partial wave corresponds to a particular description of the decay process, starting with the production of a threepion intermediate state $\mathrm{X}$ with given $J_{\mathrm{X}}^{P}$ quantum numbers. It also includes its decay into a two-pion intermediate state - the isobar $\xi$ with given $J_{\xi}^{P}$ quantum numbers - and a single $\pi^{-}$with an orbital angular momentum $L$ with respect to the isobar and the final decay of the isobar into two pions $\xi \rightarrow \pi^{+}+\pi^{-}$. The quantum numbers $J_{\mathrm{X}}^{P}$ and $J_{\xi}^{P}$ of the intermediate states $X$ and $\xi$ and the orbital angular momentum $L$ fully determine a partial wave $w$, which we will denote as

$w=\mathrm{X}[\xi \pi]_{L}$.

In our case, the $\mathrm{a}_{1}[\rho \pi]_{\mathrm{S}}$ wave will dominate the spectral intensity distribution, which corresponds to a synthesis of the $\mathrm{a}_{1}(1260)$ resonance decaying into a $\rho(770)$ and a $\pi$ in a relative $\mathrm{S}$ wave and the subsequent decay of the $\rho(770)$ into two charged pions in a relative $P$ wave.

The appearance of two undistinguishable $\pi^{-}$in the final state $\pi_{1}^{+} \pi_{2}^{-} \pi_{3}^{-}$, requires the partial wave $w$ of the hadronic current to be constructed observing Bose symmetry and thus contains two coherently summed parts:

$j_{w}^{\mu}=B_{\mathrm{X}}\left(s_{\mathrm{X}}\right)\left[B_{\xi}\left(s_{(12)}\right) t_{(12), w}^{\mu}+B_{\xi}\left(s_{(13)}\right) t_{(13), w}^{\mu}\right]$.

The real-valued tensor structures $t_{(i j), w}^{\mu}$ describe the twoparticle combination $(i j)$ to form the isobar. The complexvalued dynamic amplitudes $B_{\mathrm{X}}\left(s_{\mathrm{X}}\right)$ and $B_{\xi}\left(s_{(i j)}\right)$ describe the spectral distributions for $\mathrm{X}$ and $\xi$ with respect to their invariant mass-squares $s_{\mathrm{X}}$ and $s_{(i j)}$.

In contrast to the tensor structures $t_{(i j), w}^{\mu}$, the dynamic amplitudes cannot be derived from first principles, but have to be parameterized using existing knowledge. The particular choice of a dynamic amplitude parameterization can be 
difficult and e.g. Refs. $[5,12,13]$ use more elaborate parameterizations e.g. including a mass function for the $\mathrm{a}_{1}(1260)$. In general, the dynamic amplitude can be written as [14]:

$B_{\mathfrak{a}}(s)=\frac{n_{\mathfrak{a}}(s)}{D_{\mathfrak{a}}(s)}$,

where the real-valued $n_{\mathfrak{a}}(s)$ encodes production effects and cancels singularities in the unphysical regions at $s_{\mathfrak{a}}=0$ arising from the projection operator $g_{\mathfrak{a}}^{\mu \nu}$ [see definition in Eq. (10)]. This is possible, since the projection operator is always associated with an intermediate state $\mathfrak{a}$ and thus its dynamic amplitude $B_{\mathfrak{a}}(s)$. Its numerator $n_{\mathfrak{a}}(s)$ is often parameterized as low-order polynomial in $s$.

The denominator $D_{\mathfrak{a}}(s)$ encodes the resonance content of the dynamic amplitude and is most commonly described by a Breit-Wigner-like amplitude:

$D_{\mathfrak{a}}(s)=m_{\mathfrak{a}}^{2}-s-\mathfrak{i} m_{\mathfrak{a}} \Gamma(s)$.

They describe with a resonance mass $m_{\mathfrak{a}}$ and width $\Gamma_{\mathfrak{a}}$ an isolated resonance within a particular partial wave which lays far from any threshold. ${ }^{3}$ The mass-dependent width is hereby commonly described by:

$\Gamma(s)=\Gamma_{\mathfrak{a}}\left(\frac{q(s)}{q\left(m_{\mathfrak{a}}^{2}\right)}\right)^{2 \ell+1} \frac{m_{\mathfrak{a}}}{\sqrt{s}}$.

Here, $\ell$ is the orbital angular momentum appearing in the two body decay of the described resonance in its rest frame: $X \rightarrow \xi+\pi^{-}$or $\xi \rightarrow \pi^{+} \pi^{-}$(hereafter, the generic twobody decay will be dubbed as $\mathfrak{a} \rightarrow \mathfrak{b}+\mathfrak{c}$ ). The corresponding breakup momentum $q\left(s=m_{\mathfrak{a}}^{2}\right)$ in the decays of $\mathrm{X}$ and $\xi$ has the generic form:

$q(s)=\sqrt{\frac{s^{2}+m_{\mathfrak{b}}^{4}+m_{\mathfrak{c}}^{4}-2\left(s m_{\mathfrak{b}}^{2}+s m_{\mathfrak{c}}^{2}+m_{\mathfrak{b}}^{2} m_{\mathfrak{c}}^{2}\right)}{4 s}}$.

While Breit-Wigner-like amplitudes constitute the most common parameterizations of the resonance content, more sophisticated approaches, e.g. in Ref. [15], can also be used. Such approaches become necessary in the case of overlapping resonances or opening thresholds.

\section{Construction of hadronic tensors}

We now construct the hadronic tensors $t_{(i j), w}^{\mu}$ defined in Eq. (4) corresponding to the angular momentum quantum numbers in a given partial wave. From group theoretical considerations of the rotational group $\mathrm{SO}(3)$ one can show, that an object with orbital angular momentum quantum number $L$ is described by a tensor, which is:

\footnotetext{
${ }^{3}$ Partial-wave analyses performed in kinematic bins of $s_{\mathrm{X}}$ alleviate the necessity of a parameterization of $B_{\mathrm{X}}\left(s_{\mathrm{X}}\right)$ and allow to extract this shape from data instead.
}

1. symmetric,

2. traceless,

3. of rank $L$,

4. and transversal to the four-momentum of the decaying particle.

In the rest frame of the decaying particle, the transversality condition translates to the tensor only having space-like components. Rotations in the rest frame of a particle are governed by the rotational group $\mathrm{SO}(3)$, subgroup of the full Lorentz group, mixing only space-like components of tensors and leaving time components untouched. Thus, a tensor describing an object of non-zero spin cannot have time-like components in the corresponding rest frame. The requirements 1 and 2 are only relevant for an orbital angular momentum greater than one, since symmetry and trace are quantities defined only for tensors with rank greater than one.

We will begin with the construction of tensor amplitudes for the general two-body decay $\mathfrak{a} \rightarrow \mathfrak{b}+\mathfrak{c}$. Energy and momentum conservation requires for their four-momenta to fulfill:

$p_{\mathfrak{a}}^{\mu}=p_{\mathfrak{b}}^{\mu}+p_{\mathfrak{c}}^{\mu}$.

Based on these four-momenta we can now define the following objects ${ }^{4,5}$ :

$q_{\mathfrak{a}}^{\mu}=\frac{1}{2}\left(p_{\mathfrak{b}}^{\mu}-p_{\mathfrak{c}}^{\mu}\right) ; g_{\mathfrak{a}}^{\mu \nu}=\eta^{\mu \nu}-\frac{p_{\mathfrak{a}}^{\mu} p_{\mathfrak{a}}^{\nu}}{s_{\mathfrak{a}}} ; k_{\mathfrak{a}}^{\mu}=g_{\mathfrak{a}}^{\mu \nu} q_{\mathfrak{a}, \nu}$,

where $s_{\mathfrak{a}}=m_{\mathfrak{a}}^{2}=p_{\mathfrak{a}}^{\mu} p_{\mathfrak{a} \mu}$ and $\eta^{\mu \nu}$ is the usual Minkowski metric. $g_{\mathfrak{a}}^{\mu \nu}$ projects out the components of a four vector, which are transversal to $p_{\mathfrak{a}}^{\mu}$. Thus, $k_{\mathfrak{a}}^{\mu}$ is the only four-vector transversal to $p_{a}^{\mu}$, which we can construct.

Using the objects defined in Eq. (10), we now construct the tensor structures $\mathfrak{T}_{\mathfrak{a}}^{\mu_{1} \ldots \mu_{L}}$, which describe the decay of the particle $\mathfrak{a}$ involving an orbital angular momentum $L$, thus requiring $L$ Lorentz indices $\mu_{1}$ to $\mu_{L}$. The tensor for an Swave decay of orbital angular momentum zero $\mathfrak{T}_{\mathfrak{a}}$ is isotropic and thus simply given by unity. The first non-trivial tensor represents a $\mathrm{P}$-wave decay and is given by the components $k_{\mathfrak{a}}^{\mu}$ transversal to $p_{\mathfrak{a}}^{\mu}$ :

$\mathfrak{T}_{\mathfrak{a}}^{\mu}=k_{\mathfrak{a}}^{\mu}$,

since $k_{\mathfrak{a}}^{\mu}$ is the only vector we can construct from the available four-vectors in Eq. (9), fulfilling all requirements listed above: $k_{\mathfrak{a}}^{\mu} p_{\mathfrak{a}, \mu}=0$.

\footnotetext{
$\overline{{ }^{4} \text { In the } \mathfrak{a} \text { rest }}$ frame, $q_{\mathfrak{a}}^{\mu}=\left(q_{\mathfrak{a}}^{0}, \mathbf{q}_{\mathfrak{a}}\right)^{\top}$ with $\left|\mathbf{q}_{\mathfrak{a}}\right|=q\left(m_{\mathfrak{a}}^{2}\right)$ as defined in Eq. (8).

5 Kinematic singularities due to the factor $1 / s_{\mathfrak{a}}$ in this projector $g_{\mathfrak{a}}^{\mu \nu}$ have to be canceled by $n_{\mathfrak{a}}(s)$ in Eq. (5).
} 
The next higher order tensor of interest is $\mathfrak{T}_{\mathfrak{a}}^{\mu \nu}$ of rank two corresponding to a D-wave decay. It is given by

$\mathfrak{T}_{\mathfrak{a}}^{\mu \nu}=\frac{3}{2} k_{\mathfrak{a}}^{\mu} k_{\mathfrak{a}}^{\nu}-\frac{1}{2} g_{\mathfrak{a}}^{\mu \nu}\left(k_{\mathfrak{a}}^{\varrho} k_{\mathfrak{a}, \varrho}\right)$,

which can be shown to fulfill all requirements listed above:

$\mathfrak{T}_{\mathfrak{a}}^{\mu \nu} p_{\mathfrak{a} \mu}=0 ; \quad \mathfrak{T}_{\mathfrak{a}}^{\mu \nu}=\mathfrak{T}_{\mathfrak{a}}^{\nu \mu} ; \quad \mathfrak{T}_{\mathfrak{a}, \mu}^{\mu}=0$.

An F-wave decay is then described by the following rank 3 tensor:

$$
\begin{aligned}
\mathfrak{T}_{\mathfrak{a}}^{\mu \nu \varrho}= & \frac{5}{2} k_{\mathfrak{a}}^{\mu} k_{\mathfrak{a}}^{\nu} k_{\mathfrak{a}}^{\varrho} \\
& -\frac{1}{2}\left(k_{\mathfrak{a}}^{\sigma} k_{\mathfrak{a}, \sigma}\right)\left(k_{\mathfrak{a}}^{\mu} g_{\mathfrak{a}}^{\nu \varrho}+k_{\mathfrak{a}}^{\nu} g_{\mathfrak{a}}^{\mu \varrho}+k_{\mathfrak{a}}^{\varrho} g_{\mathfrak{a}}^{\mu \nu}\right),
\end{aligned}
$$

which again can be shown to fulfil all requirements. A recursive formula for the construction of higher orbital angular momenta $L$ tensors $\mathfrak{T}_{\mathfrak{a}}^{\mu_{1} \ldots \mu_{L}}$, with a number of $L$ Lorentz indices $\mu_{1}$ to $\mu_{L}$ can be found in Eq. ${ }^{[16]}$ (9) of Ref. [16].

\section{Axial vector currents}

Since the weak interaction violates parity, it proceeds via both vector and axial-vector currents, with $J^{P}=1^{-}$and $J^{P}=1^{+}$. Since the axial-vector current is not strictly conserved, the corresponding component in the weak interaction may give rise to contributions to the hadronic current with quantum numbers $J_{\mathrm{X}}^{P}=0^{-}$and $J_{\mathrm{X}}^{P}=1^{+}$, the latter of which is expected to dominate in the three-pion channel. Thus, we begin by constructing its contributions to the hadronic current.

Using the tensors defined in Sect. 3, we can construct the non-symmetrized hadronic currents $t_{(i j), w}^{\mu}$ for a partial wave $w$ as defined in Eq. (4) and with particles $(i j)$ forming the isobar. The most simple axial wave is $a_{1}\left[f_{0} \pi\right]_{P}$, with the $\mathrm{a}_{1}(1260)$ quantum numbers being $J_{\mathrm{a}_{1}}^{P}=1^{+}$. Here, the only non-zero angular momenta are the spin of the $\mathrm{a}_{1}(1260)$ and the orbital angular momentum $L=1$ enclosed by $\mathrm{f}_{0}$ and $\pi^{-6}$ Thus, we find:

$t_{(12), \mathrm{a}_{1}\left[\mathrm{f}_{0} \pi\right]_{\mathrm{P}}}^{\mu}=\mathfrak{T}_{\mathrm{X}}^{\mu}$,

defined in Eq. (11), with $X=a_{1}(1260)$. As the $f_{0}$ decays into two particles labeled 1 and 2, we denote the tensor in Eq. (15) with (12) such that $p_{\mathfrak{b}}^{\mu}=p_{(12)}^{\mu}$ and $p_{\mathfrak{c}}^{\mu}=p_{3}^{\mu}$, as defined in Sect. 3.

The next partial wave we discuss is $\mathrm{a}_{1}[\rho \pi]_{S}$, which describes the decay of an $\mathrm{a}_{1}(1260)$ axial vector meson into the $\rho(770)$ and $\pi^{-}$enclosing a relative $S$ wave. Here, the only non-zero angular momentum is given by the isobar spin, since

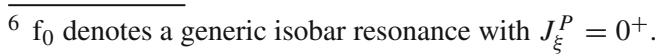

the final state pions $\pi_{1}^{+}$and $\pi_{2}^{-}$are spinless and thus their relative orbital angular momentum must equal the isobar spin. The corresponding tensor is $\mathfrak{T}_{(12)}^{\mu}$, defined in Eq. (11) with $p_{\mathfrak{b}}^{\mu}=p_{1}^{\mu}$ and $p_{\mathfrak{c}}^{\mu}=p_{2}^{\mu}$. This tensor is transversal to $p_{(12)}^{\mu}$ by definition, but not transversal to $p_{\mathrm{X}}^{\mu}$. To ensure this, we need to apply $g_{\mathrm{X}}^{\mu \nu}$, given in Eq. (10), and obtain:

$t_{(12), \mathrm{a}_{1}[\rho \pi]_{\mathrm{S}}}^{\mu}=g_{\mathrm{X}}^{\mu \nu} \mathfrak{T}_{(12), v}$.

Since the spin of the $\mathrm{a}_{1}(1260)$ and the $\rho(770)$ can also couple to $L=2$, we now also consider the partial wave $\mathrm{a}_{1}[\rho \pi]_{\mathrm{D}}$. The orbital angular momentum of $L=2$ is represented by $\mathfrak{T}_{X}^{\mu \nu}$ given in Eq. (12), with $p_{\mathfrak{b}}^{\mu}=p_{(12)}^{\mu}$ and $p_{\mathfrak{c}}^{\mu}=p_{3}^{\mu}$. The decay of $\rho(770)$ is described by the same structure as in Eq. (16) and we find for the total decay chain:

$t_{(12), \mathrm{a}_{1}[\rho \pi]_{\mathrm{D}}}^{\mu}=\mathfrak{T}_{\mathrm{X}}^{\mu \nu} \mathfrak{T}_{(12), v}$,

which is transversal to $p_{\mathrm{X}}^{\mu}$ by definition.

Finally we consider the wave $\mathrm{a}_{1}\left[\mathrm{f}_{2} \pi\right]_{\mathrm{P}}$. The appearance of an orbital angular momentum $L=1$ is described by $\mathfrak{T}_{\mathrm{X}}^{\mu}$, like in Eq. (15) and the decay of the $f_{2}(1270)$ isobar is described by $\mathfrak{T}_{(12)}^{\mu \nu}$. As discussed for the decay of the $\rho(770)$, the orbital angular momentum between $\pi_{1}^{+}$and $\pi_{2}^{-}$must match the isobar spin. Again, $\mathfrak{T}_{(12)}^{\mu \nu}$ - defined in Eq. (12) with $p_{\mathfrak{b}}^{\mu}=p_{1}^{\mu}$ and $p_{\mathfrak{c}}^{\mu}=p_{2}^{\mu}-$ is not transversal to $p_{\mathrm{X}}^{\mu}$, so we use $g_{\mathrm{X}}^{\mu \nu}$ to obtain:

$t_{(12), \mathrm{a}_{1}\left[\mathrm{f}_{2} \pi\right]_{\mathrm{P}}}^{\mu}=g_{\mathrm{X}}^{\mu \nu} \mathfrak{T}_{(12), \nu \varrho} \mathfrak{T}_{(12)}^{\varrho}$.

For a general amplitude with arbitrary $L$ we need an isobar spin of $J_{\xi}=L \pm 1$ to be able to construct a spin 1 tensor. For $L=J_{\xi}+1$ we find

$t_{(12), \mathrm{a}_{1}\left[\xi_{j} \pi\right]_{L}}^{\mu}=\mathfrak{T}_{\mathrm{X}}^{\mu \nu_{1} \ldots \nu_{J_{\xi}}} \mathfrak{T}_{(12), \nu_{1} \ldots \nu_{J_{\xi}}}$,

since the only non-contracted Lorentz index $\mu$ comes from $\mathfrak{T}_{\mathrm{X}}^{\mu v_{1} \ldots v_{J_{\xi}}}$, which is already transversal to $p_{\mathrm{X}}^{\mu}$. This is not the case for $L=J_{\xi}-1$, where we need to apply the projection operator and find:

$t_{(12), \mathrm{a}_{1}\left[\xi_{j} \pi\right]_{L}}^{\mu}=g_{\mathrm{X}}^{\mu \nu} \mathfrak{T}_{(12), v \varrho_{1} \ldots \varrho_{L}} \mathfrak{T}_{\mathrm{X}}^{\varrho_{1} \ldots \varrho_{L}}$.

For the Bose symmetrized tensors $t_{(13), a}^{\mu}$, defined in Eq. (4), the four-momenta $p_{2}^{\mu}$ and $p_{3}^{\mu}$ are interchanged in all formulas. Even though we only explicitly mentioned the ground-state $\mathrm{a}_{1}(1260)$ resonance and the $\rho(770)$ and $\mathrm{f}_{2}(1270)$ isobars, the formulas in this section are equally valid for all excited $\mathrm{a}_{1}^{\prime}, \rho^{\prime}, \mathrm{f}_{0}^{\prime}$ and $\mathrm{f}_{2}^{\prime}$ resonances.

\section{Vector currents}

Since the vector current is conserved, the vector component of the weak interaction can only give rise to a hadronic vector current with quantum numbers $J_{\mathrm{X}}^{P}=1^{-}$and not $J_{\mathrm{X}}^{P}=$ 
$0^{+}$. Owing to $G$-parity, the hadronic vector current is only expected to contribute to final states with an even number of final-state pions, while its contributions to the three-pion final state is usually assumed to vanish. In this section, we nevertheless give the expected dominant contribution to a hadronic three-pion vector current to be able to explicitly search for isospin violation.

The most prominent example of this is the $\pi_{1}[\rho \pi]_{\mathrm{P}}$ wave, which involves the spin exotic state $\pi_{1}(1600)$ with $J_{\pi_{1}}^{P}=1^{-}$. In this wave, the isobar spin and the orbital angular momentum are equal $J_{\xi}=L=1$ and thus we cannot obtain a tensor of spin 1 by simple contraction of Lorentz indices as in Sect. 4. Using the totally antisymmetric tensor $\varepsilon^{\mu \nu \varrho \sigma}$, we construct:

$t_{(12), \pi_{1}[\rho \pi]_{\mathrm{P}}}^{\mu}=\varepsilon^{\mu \nu \varrho \sigma} p_{\mathrm{X}, \nu} \mathfrak{T}_{\mathrm{X}, \varrho} \mathfrak{T}_{(12), \sigma}$,

which is transversal to $p_{\mathrm{X}}^{\mu}$ by definition, due to the permutation properties of $\varepsilon^{\mu \nu \varrho \sigma}$. The tensors of orbital angular momentum 1, $\mathfrak{T}_{X}^{\mu}$ and $\mathfrak{T}_{(12)}^{\mu}$, are given in Eqs. (15) and (16). Due to the antisymmetric tensor $\varepsilon^{\mu \nu \varrho \sigma}$, the tensor $t_{(12), \pi_{1}[\rho \pi]_{\mathrm{P}}}^{\mu}$ transforms differently under the parity operator than the tensors constructed in Sect. 4 and therefore the corresponding three-pion state is of type $\pi_{1}$ instead of type $a_{1}$.

For the construction of vector currents involving higher isobar spins $J_{\xi}$ and angular momenta $L$, both must be equal and all but one of the Lorentz indices on both corresponding tensors must be contracted with one another. The single remaining index of both tensors is contracted with $\varepsilon^{\mu \nu \varrho \sigma}$, similar to Eq. (21).

\section{Current for pseudo-scalars}

As mentioned in Sect. 4, the non-conservation of the axialvector current in the weak interaction also allows pseudoscalar resonances with $J_{\mathrm{X}}^{P}=0^{-}$to contribute to the hadronic current. In analogy to the decay of $\tau$ leptons into a single pion, which also carries $J_{\pi}^{P}=0^{-}$, the pseudo-scalar hadronic current is simply given by $p_{\mathrm{X}}^{\mu}$ multiplied by a Lorentz scalar $t_{(12), \pi^{\prime}[\xi \pi]_{L}}$ :

$t_{(12), \pi^{\prime}[\xi \pi]_{L}}^{\mu}=p_{\mathrm{X}}^{\mu} t_{(12), \pi^{\prime}[\xi \pi]_{L}}$,

where the spin $J_{\xi}$ of the isobar and $L$ must be equal. The scalar factor $t_{(12), \pi^{\prime}[\xi \pi]_{L}}$ for the decay of a pseudo-scalar X via an isobar $\xi$ can be constructed from the same tensors, as the axial and vector currents:

$t_{(12), \pi^{\prime}[\xi \pi]_{L}}=\mathfrak{T}_{\mathrm{X}}^{\mu_{1} \ldots \mu_{L}} \mathfrak{T}_{(12), \mu_{1} \ldots \mu_{L}}$.

Note, that in contrast to axial and vector currents, this pseudo scalar current does not have to be transversal to $p_{\mathrm{X}}^{\mu}$, since it must be invariant under rotations in the $\mathrm{X}$ rest frame and therefore can have only a time component.

\section{Scope and limitations of the isobar model}

The amplitudes derived here are all formulated within the isobar model, i.e. the assumptions of two subsequent twoparticle decays producing the three-body final state. This isobar model [9] is the reliable tool for amplitude analyses for three-body final states and is used in all analyses relating kinematic information to $J^{P C}$ decompositions. The underlying assumptions have shown to be valid $[17,18]$. In addition, the isobar model can be extended by waves of given $J^{P C}$ quantum numbers without pre-imposing a specific resonance content $[17,18]$.

Apart from a model fully formulated within the isobar model, the amplitudes developed here can be used to extend alternative models, like resonance chiral theory, to include contributions from resonances with higher mass or spin, like $f_{2}(1270)$ or $a_{1}(1420)$ which are typically absent from such approaches [19].

\section{Connection to form factors}

We may also use a formulation for the hadronic current, which is complementary to Eq. (2), namely an expansion in terms of the four four-vectors constructed from the fourmomenta of the final state particles. This expansion introduces complex-valued form factors to describe the hadronic structure. These four-vectors are:

$$
\begin{aligned}
p_{i \perp}^{\mu} & =g_{\mathrm{X}}^{\mu \nu} p_{i, v} \\
p_{\varepsilon}^{\mu} & =\varepsilon^{\mu \nu \lambda \sigma} p_{1, \nu} p_{2, \lambda} p_{3, \sigma} \\
p_{\mathrm{X}}^{\mu} & =p_{1}^{\mu}+p_{2}^{\mu}+p_{3}^{\mu},
\end{aligned}
$$

where $p_{i}^{\mu}$ are the pion-momenta and $g_{\mathrm{X}}^{\mu \nu}$ is defined in Eq. (10). Thus, $p_{i \perp}^{\mu}$ are the components of $p_{i}^{\mu}$ that are transversal to $p_{\mathrm{X}}^{\mu}$. Using these, the expansion of the hadronic current in terms of form factors $F_{v}$ reads:

$J_{\text {had }}^{\mu}=p_{2 \perp}^{\mu} F_{2}+p_{3 \perp}^{\mu} F_{3}+p_{\varepsilon}^{\mu} F_{\varepsilon}+p_{\mathrm{X}}^{\mu} F_{\mathrm{s}}$,

where $F_{2}$ and $F_{3}$ are two axial form-factors, $F_{\varepsilon}$ is the vector form-factor, and $F_{\mathrm{S}}$ is the scalar from factor. The form factors are functions of the Lorentz invariant quantities $\left\{s_{\mathrm{X}}, s_{(12)}, s_{(13)}, s_{(23)}, m_{\pi}^{2}\right\}$. Similar decompositions into form factors of the hadronic current have already been given in Refs. [5,8] to which we relate our results in Sect. 9.

Since there is only one four-vector for each of the scalar and vector form-factors with the respective transformation properties, only a single scalar form-factor and a single vector form-factor appear. There are two independent axial formfactors, since there are only two linearly independent corresponding four-vectors due to momentum conservation in the $\mathrm{X}$ rest frame: 
$p_{1 \perp}^{\mu}=-p_{2 \perp}^{\mu}-p_{3 \perp}^{\mu}$.

Analogue to the hadronic current, we can expand the form factors into a series of partial waves:

$$
\begin{aligned}
F_{v}= & \sum_{w \in \text { waves }} \mathcal{C}_{w} B_{\mathrm{X}}\left(s_{\mathrm{X}}\right)\left[B_{\xi}\left(s_{(12)}\right) f_{v, w}^{(12)}\right. \\
& \left.+B_{\xi}\left(s_{(13)}\right) f_{v, w}^{(13)}\right] .
\end{aligned}
$$

The sum extends over the sub-set of partial waves $w$ with $J_{\mathrm{X}}^{P}$ quantum numbers matching the corresponding $F_{v}$. Partialwave form-factors $f_{v, w}^{(i j)}$ encode the contribution of partial wave $w$ to the form factor $v \in\{2,3, \varepsilon, \mathrm{s}\}$. ( $i j)$ denote the final-state particles forming the isobar. In the following, we will match the tensor expressions derived in Sects. 4-6 to the corresponding form factors in Eq. (27).

\subsection{Axial form factors}

Here, we discuss the expansion of the axial currents from Sect. 4 for a partial wave $w$ in terms of partial-wave formfactors $f_{v, w}^{(12)}$, such that:

$t_{(12), w}^{\mu}=p_{2 \perp}^{\mu} f_{2, w}^{(12)}+p_{3 \perp}^{\mu} f_{3, w}^{(12)}$.

For the axial-vector like waves discussed in Sect. 4, we derive the corresponding form factors by performing all Lorentzcontractions given in Sect. 4:

$f_{2, \mathrm{a}_{1}\left[\mathrm{f}_{0} \pi\right]_{\mathrm{P}}}^{(12)}=0 ; \quad f_{3, \mathrm{a}_{1}\left[\mathrm{f}_{0} \pi\right]_{\mathrm{P}}}^{\left(\mathrm{p}_{\mathrm{P}}\right)}=-1$

and

$f_{2, \mathrm{a}_{1}[\rho \pi]_{\mathrm{S}}}^{(12)}=-1 ; \quad f_{3, \mathrm{a}_{1}[\rho \pi]_{\mathrm{S}}}^{(12)}=-\frac{1}{2}$.

For the $\mathrm{a}_{1}[\rho \pi]_{\mathrm{D}}$ wave, the respective form factors are:

$$
\begin{aligned}
f_{2, \mathrm{a}_{1}[\rho \pi]_{\mathrm{D}}}^{(12)}= & \frac{1}{8}\left(2 s_{(12)}-s_{\mathrm{X}}+2 m_{\pi}^{2}\right)-\frac{\left(s_{(12)}-m_{\pi}^{2}\right)^{2}}{8 s_{\mathrm{X}}} ; \\
f_{3, \mathrm{a}_{1}[\rho \pi]_{\mathrm{D}}}^{(12)}= & \frac{3}{16}\left(s_{(13)}-s_{(23)}\right)\left(1+\frac{s_{(12)}-m_{\pi}^{2}}{s_{\mathrm{X}}}\right) \\
& +f_{2, \mathrm{a}_{1}[\rho \pi]_{\mathrm{D}}}^{(12)} / 2 .
\end{aligned}
$$

And for $\mathrm{a}_{1}\left[\mathrm{f}_{2} \pi\right]_{\mathrm{P}}$ :

$$
\begin{aligned}
f_{2, \mathrm{a}_{1}\left[\mathrm{f}_{2} \pi\right]_{\mathrm{P}}}^{(12)}= & \frac{3}{16}\left(s_{(13)}-s_{(23)}\right)\left(1+\frac{s_{(12)}-m_{\pi}^{2}}{s_{\mathrm{X}}}\right) ; \\
f_{3, \mathrm{a}_{1}\left[\mathrm{f}_{2} \pi\right]_{\mathrm{P}}}^{(12)}= & \frac{4 m_{\pi}^{2}-s_{(12)}}{32 s_{\mathrm{X}} s_{(12)}}\left(s_{\mathrm{X}}+s_{(12)}-m_{\pi}^{2}\right)^{2} \\
& +f_{2, \mathrm{a}_{1}\left(\mathrm{f}_{2} \pi\right)_{\mathrm{P}}}^{(12)} / 2 .
\end{aligned}
$$

Note, that Eqs. (30)-(32) are only valid in the case of identical final state masses ${ }^{7} m_{i}^{2}=p_{i}^{\mu} p_{i, \mu}=m_{\pi}^{2}$, while the results of Sect. 4 are valid in full generality. For arbitrary final-state masses, we give the form factors in Sect. A. The Bose symmetrized axial form-factors are obtained as:

$f_{2, w}^{(13)}=\left.f_{3, w}^{(12)}\right|_{s_{(12)} \leftrightarrow s_{(13)}} \quad$ and $\quad f_{3, w}^{(13)}=\left.f_{2, w}^{(12)}\right|_{s_{(12)} \leftrightarrow s_{(13)}}$

In Sect. A, we also give form factors for the $\mathrm{a}_{1}\left[\mathrm{f}_{2} \pi\right]_{\mathrm{F}}$ and the $\mathrm{a}_{1}\left[\rho_{3} \pi\right]_{\mathrm{D}}$ waves, the latter containing the spin 3 resonance $\rho_{3}(1690)$.

\subsection{Vector form-factor}

For the vector current, we can write the tensor amplitude derived in Sect. 5 as:

$t_{(i j), \pi_{1}[\rho \pi]_{\mathrm{P}}}^{\mu}=p_{\varepsilon}^{\mu} f_{\varepsilon, \pi_{1}[\rho \pi]_{\mathrm{P}}}^{(i j)}$,

for which we find

$f_{\varepsilon, \pi_{1}[\rho \pi]_{\mathrm{P}}}^{(12)}=1 ; \quad f_{\varepsilon, \pi_{1}[\rho \pi]_{\mathrm{P}}}^{(13)}=-1$.

\subsection{Scalar form-factors}

Since the scalar part of the hadronic current already contains the desired structure for the decomposition into form-factors we simply identify $f_{\mathrm{s}, w}^{(12)}=t_{(12), w}$ defined in Eq. (22). Here, we give explicit expressions for $\pi^{\prime}\left[f_{0} \pi\right]_{S}$ and $\pi^{\prime}[\rho \pi]_{\mathrm{P}}$ :

$f_{\mathrm{S}, \pi^{\prime}\left[\mathrm{f}_{0} \pi\right]_{\mathrm{S}}}^{(12)}=1$

and

$f_{\mathrm{S}, \pi^{\prime}[\rho \pi]_{\mathrm{P}}}^{(12)}=\frac{1}{8}\left(s_{(23)}-s_{(13)}\right)\left(1+\frac{s_{(12)}-m_{\pi}^{2}}{s_{\mathrm{X}}}\right)$.

Note, that Eq. (37) again only holds for equal final-state masses. The formulas for arbitrary final state masses and for the $\pi^{\prime}\left[\mathrm{f}_{2} \pi\right]_{\mathrm{D}^{-}}$and $\pi^{\prime}\left[\rho_{3} \pi\right]_{\mathrm{F}}$-waves are given in Sect. A. The Bose symmetrized scalar form-factors are:

$f_{\mathrm{s}, w}^{(13)}=\left.f_{\mathrm{s}, w}^{(12)}\right|_{s_{(12)} \leftrightarrow s_{(13)}}$

\section{Comparison with previous work}

In this section, we relate our findings to previously published work in Refs. [5,8].

In Ref. [8], the decomposition of the hadronic currents is given in Eq. ${ }^{[8]}$ (3), using four form factors labeled $F_{i}^{[8]}$ modifying the vectors $V_{i}^{[8] \mu}$ built from a combination of the

$\overline{7 \text { We used } p_{i}^{\mu}} p_{j}^{\mu}=\frac{s_{(i j)}-2 m_{\pi}^{2}}{2}$ in the calculation. 
four momenta of the outgoing three-particle state. ${ }^{8}$ The vector $V_{4}^{[8] \mu}$ corresponds to our $p_{\mathrm{X}}^{\mu}$ and we can identify $F_{4}^{[8]}$ with our scalar form-factor $F_{\mathrm{s}}$.

The ordering of final-state particles in Ref. [8] is given by $\pi_{1}^{-} \pi_{2}^{-} \pi_{3}^{+}$and thus we identify the corresponding momenta $q_{i}^{[8] \mu}$ with our momenta $p_{i}^{\mu}$ such, that:

$q_{1}^{[8] \mu}=p_{2}^{\mu} ; \quad q_{2}^{[8] \mu}=p_{3}^{\mu} ; \quad q_{3}^{[8] \mu}=p_{1}^{\mu}$.

This corresponds to two permutations in the definition of $V_{3}^{[8] \mu}$ as compared to Eq. (24). We can identify the vector form-factor as $F_{\varepsilon}=i F_{3}^{[8]}$. The remaining vectors in Eq. ${ }^{[8]}$ (3) of Ref. [8] are:

$V_{1}^{[8] \mu}=p_{2 \perp}^{\mu}-p_{1 \perp}^{\mu} ; \quad V_{2}^{[8] \mu}=p_{3 \perp}^{\mu}-p_{1 \perp}^{\mu}$.

Using Eq. (26), we can relate our axial form-factors $F_{2}$ and $F_{3}$ to the axial form-factors $F_{1}^{[8]}$ and $F_{2}^{[8]}$ of Ref. [8], as follows:

$F_{2}=2 F_{1}^{[8]}+F_{2}^{[8]} ; \quad F_{3}=F_{1}^{[8]}+2 F_{2}^{[8]}$.

In Ref. [5], the decomposition of the hadronic current is given in Eq. ${ }^{[5]}$ (A2) of Ref. [5]. The authors of Ref. [5] do not eliminate one of the axial form-factors using Eq. (26) and thus use a total of five form factors. Ref. [5] discusses the decay into $\pi_{1}^{0} \pi_{2}^{0} \pi_{3}^{-}$, thus we translate it to the $3 \pi^{ \pm}$channel using the particle ordering $\pi_{1}^{-} \pi_{2}^{-} \pi_{3}^{+}$, consistent with Ref. [8].

Using this ordering, we identify:

$F_{\mathrm{S}}=c_{4} F_{4}^{[5]} ; \quad F_{\varepsilon}=i c_{5} F_{5}^{[5]}$.

The axial part $J_{\text {axial }}^{\mu}$ of the hadronic current of Ref. [5] can be written as:

$$
\begin{aligned}
J_{\text {axial }}^{\mu}= & c_{1}\left(p_{3 \perp}^{\mu}-p_{1 \perp}^{\mu}\right) F_{1}^{[5]} \\
& +c_{2}\left(p_{1 \perp}^{\mu}-p_{2 \perp}^{\mu}\right) F_{2}^{[5]}+c_{3}\left(p_{2 \perp}^{\mu}-p_{3 \perp}^{\mu}\right) F_{3}^{[5]} .
\end{aligned}
$$

And we identify the form factors $F_{2}$ and $F_{3}$ from Eq. (26):

$$
\begin{aligned}
& F_{2}=c_{1} F_{1}^{[5]}-2 c_{2} F_{2}^{[5]}+c_{3} F_{3}^{[5]} \\
& F_{3}=2 c_{1} F_{1}^{[5]}-c_{2} F_{2}^{[5]}-c_{3} F_{3}^{[5]} .
\end{aligned}
$$

The Lorentz invariant quantities contained in the form factors match as:

$s_{1}=s_{(13)} ; \quad s_{2}=s_{(12)} ; \quad s_{3}=s_{(23)}$.

We also compare the results for the individual partial wave contributions to the hadronic current given in Sect. 4 above with the expressions given in Eq. ${ }^{[5]}(\mathrm{A} 3)$. First, we note that

\footnotetext{
8 Since our work uses similar notation to previous work, we annotate quantities defined outside this article with the respective literature reference to avoid confusion.
}

Ref. [5] does not include a parameterization for the dynamic amplitude of the $3 \pi$ state $X$, since the analysis was performed independently in mass bins of $\mathrm{X}, m_{3 \pi}=\sqrt{s_{\mathrm{X}}}$. Second, in the dynamic amplitudes in Ref. [5] an additional factor $F_{R j}\left(k_{k}\right)$ multiplies the isobar Breit-Wigner. ${ }^{9}$ Since this real-valued factor only depends on the invariant masses of $\mathrm{X}$ and $\xi$, it can be absorbed into $n_{\mathfrak{a}}(s)$ of Eq. (5). As stated in Sect. 2, we do not discuss this factor.

Finally, we compare the tensor structures for the individual partial waves. The mathematical formulation of the amplitudes corresponds to the $\pi^{0} \pi^{0} \pi^{-}$final state and thus Bose symmetrization is already explicitly written in Ref. [5] for $\rho$-like isobars, since these can be formed by both possible $\left(\pi^{-} \pi^{0}\right)$ systems. However, no symmetrization is needed for $\mathrm{f}_{0}$-like and $\mathrm{f}_{2}$-like isobars, since these can only be formed by $\left(\pi^{0} \pi^{0}\right)$. For our final state $\pi^{+} \pi^{-} \pi^{-}$, all isobars appear with a Bose symmetrization term.

We now compare the Bose-symmetrization terms for the individual partial waves. For the $a_{1}\left[f_{0} \pi\right]_{P}$ wave, which corresponds to waves 6 and 7 in Ref. [5] our results agree with Eq. ${ }^{[5]}(\mathrm{A} 3)$.

For the $\mathrm{a}_{1}[\rho \pi]_{\mathrm{S}}$ waves, which correspond to waves 1 and 2 in Ref. [5], we only agree in case of equal-mass mesons forming the isobar. Thus, for $\pi^{0} \pi^{0} \pi^{-}$a small deviation is introduced, owing to the difference between $m_{\pi^{ \pm}}$and $m_{\pi^{0}}$. The origin of this deviation is a missing projection operator $g_{(12)}^{\mu \nu}$, as defined in Eq. (10), coming from the $\rho(770)$ propagator [21].

For $\mathrm{a}_{1}[\rho \pi]_{\mathrm{D}}$ waves, there is a discrepancy with respect to the corresponding waves 3 and 4 of Ref. [5]. This might not be surprising at first sight, as their amplitudes do not correspond to $L$ eigenstates, but to two Born term amplitudes. Using the naming scheme of Eq. (3), replacing the upper case letters for $L$ by lower case letters and thus following Ref. [5], we can write these Born term amplitudes as given in Eq. ${ }^{[21]}$ (1) of Ref. [21]:

$t_{(12), \mathrm{a}_{1}[\rho \pi]_{s}}^{\mu}=g_{\mathrm{X}}^{\mu \nu} \eta_{\nu \varrho} k_{(12)}^{\varrho}$,

$t_{(12), \mathrm{a}_{1}[\rho \pi]_{d}}^{\mu}=g_{\mathrm{X}}^{\mu \nu} p_{(12), v} p_{\mathrm{X} \varrho} k_{(12)}^{\varrho}$.

Ref. [21] states, that every linear combination of these two Born term amplitudes with Lorentz scalar coefficients constitutes a valid amplitude for the decay $\tau \rightarrow 3 \pi^{ \pm}+v$. Indeed, we can write the amplitudes corresponding to $L$ eigenstates as such linear combinations of Born term amplitudes. For

\footnotetext{
$\overline{9}$ The nominal fit of Ref. [5] has $F_{R j}\left(k_{k}\right)=1$, making it equal to our approach.
} 
our D-wave amplitude we find ${ }^{10}$ :

$$
\begin{aligned}
t_{(12), \mathrm{a}_{1}[\rho \pi]_{\mathrm{D}}}^{\mu}= & c_{s}\left(s_{\mathrm{X}}, s_{(12)}\right) t_{(12), \mathrm{a}_{1}[\rho \pi]_{s}}^{\mu} \\
& +c_{d}\left(s_{\mathrm{X}}, s_{(12)}\right) t_{(12), \mathrm{a}_{1}[\rho \pi]_{d}}^{\mu},
\end{aligned}
$$

where $c_{s}\left(s_{\mathrm{X}}, s_{(12)}\right)$ and $c_{d}\left(s_{\mathrm{X}}, s_{(12)}\right)$ are two Lorentz-scalar coefficients that, however, depend on $s_{\mathrm{X}}$ and $s_{(12)}$. We can invert this to obtain the Born term amplitudes as linear combination of the amplitudes describing $L$ eigenstates in an analogue way:

$$
\begin{aligned}
t_{(12), \mathrm{a}_{1}[\rho \pi]_{d}}^{\mu}= & -\frac{c_{s}\left(s_{\mathrm{X}}, s_{(12)}\right)}{c_{d}\left(s_{\mathrm{X}}, s_{(12)}\right)} t_{(12), \mathrm{a}_{1}[\rho \pi]_{\mathrm{S}}}^{\mu} \\
& +\frac{1}{c_{d}\left(s_{\mathrm{X}}, s_{(12)}\right)} t_{(12), \mathrm{a}_{1}[\rho \pi]_{\mathrm{D}}}^{\mu} .
\end{aligned}
$$

We now insert the Born term $\mathrm{a}_{1}[\rho \pi]_{d}$ tensor structure into Eq. (4) to construct the corresponding partial-wave hadroniccurrent within the isobar model and find using Eq. (50):

$$
\begin{aligned}
j_{\mathrm{a}_{1}[\rho \pi]_{d}}^{\mu}= & B_{\mathrm{a}_{1}}\left(s_{\mathrm{X}}\right) B_{\rho}\left(s_{(12)}\right)\left(-\frac{c_{s}\left(s_{\mathrm{X}}, s_{(12)}\right)}{c_{d}\left(s_{\mathrm{X}}, s_{(12)}\right)} t_{(12), \mathrm{a}_{1}[\rho \pi]_{\mathrm{S}}}^{\mu}\right. \\
& \left.+\frac{1}{c_{d}\left(s_{\mathrm{X}}, s_{(12)}\right)} t_{(12), \mathrm{a}_{1}[\rho \pi]_{\mathrm{D}}}^{\mu}\right)+ \text { Bose symm. }(51
\end{aligned}
$$

The coefficients in Eq. (50), which depend on $s_{\mathrm{X}}$ and $s_{(12)}$, now result in additional terms multiplying the partial-wave hadronic-currents in Eq. (51), expressed in the $L$-eigenstate basis. Thus, the naive use of Born term amplitudes within the context of the isobar model results in effective distortions of the dynamic isobar amplitudes $B_{\mathrm{a}_{1}}\left(s_{\mathrm{X}}\right)$ and $B_{\rho}\left(s_{(12)}\right)$.

We also find discrepancies for the $\mathrm{a}_{1}\left[\mathrm{f}_{2} \pi\right]_{\mathrm{P}}$ wave, again resulting in an $s_{\mathrm{X}}$ and $s_{(12)}$ dependent factor, which can be absorbed into the dynamic amplitudes, leading to distortions in the corresponding two-hadron dynamic amplitude. We attribute the discrepancy to a missing projector for the $\mathrm{P}$ wave tensor, which is due to the amplitudes of Ref. [5] not corresponding to $L$-eigenstates. And as Ref. [5] does not include the $\mathrm{a}_{1}\left[\mathrm{f}_{2} \pi\right]_{f}$, amplitudes for $L$ eigenstates cannot be constructed as linear combinations.

Finally, additional discrepancies between our approach and Ref. [5] appear for the case of final-states formed by nonequal mass particles for the $\mathrm{a}_{1}[\rho \pi]_{\mathrm{D}^{-}}$wave and the $\mathrm{a}_{1}\left[\mathrm{f}_{2} \pi\right]_{\mathrm{P}^{-}}$ wave. This is again due to a missing projection operator for the isobar, as in the $\mathrm{a}_{1}[\rho \pi]_{\mathrm{S}}$-wave.

\footnotetext{
10 The $L$ eigenstates in Ref. [21] correspond to the ones in the helicity formalism e.g. layed out in Ref. [22], while we use the covariant tensor formalism described in Ref. [16]. The connection between both formalisms can be found in Ref. [23].
}

\section{Summary and discussion}

In this article, we introduced the general formalism to describe the hadronic current in semileptonic $\tau$ decays within the framework of the isobar model. We explicitly constructed individual partial wave contributions using the decay $\tau^{-} \rightarrow$ $3 \pi^{ \pm}+v$ as an example.

For this formalism, we included contributions from partial waves with orbital angular momenta up to three units of $\hbar$, which had not been accounted for in previous works. In addition to contributions from axial-vector currents, we also allowed for contributions from vector and pseudo-scalar resonances, which were assumed to vanish in Refs. [5,8]. These additional contributions allow for the search of pseudo-scalar $3 \pi$ resonances, like the $\pi(1300)$, and of vector resonances, like the $\pi_{1}(1600)$ in $\tau$ decays.

We then translated the individual contributions of the partial-wave hadronic currents into the formalism of form factors and compared to previous results from Refs. [5,8].

Finally, we confront our results for the individual partial wave currents to the ones quoted in Ref. [5] and we find two sources of discrepancies. First, it is important to note that the waves in Ref. [5] are not describing $L$-eigenstates and thus do not trivially translate to the isobar model, as they cause distortions of the dynamic amplitudes. Second, the waves in Ref. [5] do not project out the transversal components of the tensor structure describing the isobar decay. This leads to discrepancies with respect to our approach for the case of final states with non-equal mass particles.

We want to state, that an explicit construction of partial waves with given quantum numbers is necessary to make results interpretable in terms of the hadronic excitation spectrum. A fit with partial waves with mixed quantum numbers and distorted dynamic amplitudes does not give reliable information on the physics sorted by orbital angular momentum quantum numbers in $\tau$ decays.

The expressions developed here allow for the first time to extend partial-wave analyses of the decay $\tau \rightarrow 3 \pi^{-}+v_{\tau}$, by including previously neglected contributions: higher orbital angular momenta with $L>2$, as well as pseudo-scalar and vector contributions.

Beyond the study of the hadronic spectrum itself, a proper description of the decay kinematics of the decay $\tau \rightarrow 3 \pi^{ \pm}+$ $v$ is necessary for the precision measurements of $\tau$ properties like the electric dipole moment (EDM). For this, we have to correlate the decay distributions of both $\tau^{ \pm}$leptons in $\tau$-pair production. For the measurement of an EDM we single out $\mathrm{CP}$ odd correlations and search for $\mathrm{CP}$ violations therein. We thereby rely on maximal sensitivity to the spin correlation of the $\tau^{ \pm}$pair. The necessary spin analyzing power is best obtained from hadronic final states [20]. If we use threepion final states we need to know the contributions of the different partial waves for we have to precisely describe the 
$\tau \rightarrow 3 \pi+v$ decay distribution. Using three pions in the final state, we rely on the proper description of the $\tau \rightarrow 3 \pi^{ \pm}+v$ decay kinematics reflecting the contributions of the partial waves.

We have investigated the sensitivity of the value for the EDM extracted from pseudo data on the description used for the $3 \pi$ final state. For this, we have first generated pseudo data using various input values for the EDM and then analyzed the data using the method from Ref. [20]. For the analysis, we assumed a variety of partial wave compositions and determined the deviation of the extracted EDM values from the input values. In particular, the use of a wrong hadronic model leads to an under-estimation of the EDM by a factor of up to two. We found these deviations to depend on the overlap between the input hadronic model and the model used in the analysis. We thus demonstrated that a false EDM may be extracted from data, which is generated through an incomplete understanding of the hadronic final states.

Acknowledgements We thank M. Mikhasenko for useful discussions on the proper parameterization of resonance amplitudes.

Data Availability Statement This manuscript has no associated data or the data will not be deposited. [Authors' comment: The manuscript gives the framework for future detailed partial-wave analysis of threebody semileptonic tau decays. As such, there is no data associated yet to be deposited.]

Open Access This article is licensed under a Creative Commons Attribution 4.0 International License, which permits use, sharing, adaptation, distribution and reproduction in any medium or format, as long as you give appropriate credit to the original author(s) and the source, provide a link to the Creative Commons licence, and indicate if changes were made. The images or other third party material in this article are included in the article's Creative Commons licence, unless indicated otherwise in a credit line to the material. If material is not included in the article's Creative Commons licence and your intended use is not permitted by statutory regulation or exceeds the permitted use, you will need to obtain permission directly from the copyright holder. To view a copy of this licence, visit http://creativecomm ons.org/licenses/by/4.0/.

Funded by SCOAP ${ }^{3}$.

\section{A Form factors for arbitrary final-state masses}

To complete the discussion in Sect. 8, we extend the formulae for the form factors to three-hadron final states with arbitrary masses, $m_{1}, m_{2}$ and $m_{3}$. The isobar shall be formed by particles 1 and 2, as denoted in (12). The form factors for the wave $a_{1}\left[f_{0} \pi\right]_{P}$ are described in Eq. (29). To obtain the proper expression for $\mathrm{a}_{1}[\rho \pi]_{\mathrm{S}}$ we express all Lorentz contractions in the tensor formalism (see Sect. 3) by Lorentz invariants:

$$
f_{2, \mathrm{a}_{1}[\rho \pi]_{\mathrm{S}}}^{(12)}=-1 ; \quad f_{3, \mathrm{a}_{1}[\rho \pi]_{\mathrm{S}}}^{(12)}=-\frac{1}{2}\left(1-\frac{m_{1}^{2}-m_{2}^{2}}{s_{(12)}}\right)
$$

In order to extend the formulation of form factors for partial waves involving higher oribital angular momenta, we define the following expressions for convenience:

$$
\begin{aligned}
\mathrm{FI}= & -s_{\mathrm{X}}^{2}-\left(m_{3}^{2}-s_{(12)}\right)^{2}+2 s_{\mathrm{X}}\left(s_{(12)}+m_{3}^{2}\right) \\
\mathrm{FII}= & \left(s_{\mathrm{X}}+s_{(12)}-m_{3}^{2}\right)\left(m_{2}^{2} m_{3}^{2}+s_{(12)}\left(m_{2}^{2}+m_{3}^{2}\right)\right. \\
& -s_{(12)}^{2}+s_{\mathrm{X}}\left(s_{(12)}-m_{2}^{2}\right) \\
& \left.+m_{1}^{2}\left(s_{\mathrm{X}}-m_{3}^{2}+s_{(12)}\right)-2 s_{(12)} s_{(13)}\right) \\
\mathrm{FIII}= & s_{\mathrm{X}} s_{(12)}\left(m_{1}^{2}-m_{2}^{2}\right)^{2} \\
& -s_{\mathrm{X}} s_{(12)}^{2}\left(2 m_{1}^{2}+2 m_{2}^{2}-s_{(12)}\right) \\
\mathrm{FIV}= & m_{1}^{4}+\left(s_{(12)}-m_{2}^{2}\right)^{2}-2 m_{1}^{2}\left(s_{(12)}+m_{2}^{2}\right) \\
\mathrm{FV}= & \left(s_{\mathrm{X}}-m_{3}^{2}\right)\left(m_{1}^{2}-m_{2}^{2}\right) \\
& -s_{(12)}\left(s_{(13)}-s_{(23)}\right) .
\end{aligned}
$$

For the $\mathrm{a}_{1}[\rho \pi]_{\mathrm{D}}$ wave, $f_{2, \mathrm{a}_{1}[\rho \pi]_{\mathrm{D}}}^{(12)}$ stays unchanged and is given in Eq. (31), where $m_{\pi}^{2}$ is substituted with $m_{3}^{2}$. For $f_{3, \mathrm{a}_{1}[\rho \pi]_{\mathrm{D}}}^{(12)}$ we find:

$$
\begin{aligned}
f_{3, \mathrm{a}_{1}[\rho \pi]_{\mathrm{D}}}^{(12)}= & \frac{1}{16 s_{\mathrm{X}} s_{(12)}}\left[\left(s_{(12)}-m_{1}^{2}+m_{2}^{2}\right) \mathrm{FI}\right. \\
& \left.-3\left(s_{\mathrm{X}}-m_{3}^{2}+s_{(12)}\right) \mathrm{FV}\right]
\end{aligned}
$$

For the generalized version of the $\mathrm{a}_{1}\left[\mathrm{f}_{2} \pi\right]_{\mathrm{P}}$ form factors, we find:

$f_{2, \mathrm{a}_{1}\left[\mathrm{f}_{2} \pi\right]_{\mathrm{P}}}^{(12)}=-3 \frac{s_{\mathrm{X}}+s_{(12)}-m_{3}^{2}}{16 s_{\mathrm{X}} s_{(12)}} \mathrm{FV}$

and

$$
\begin{aligned}
f_{3, \mathrm{a}_{1}\left[\mathrm{f}_{2} \pi\right]_{\mathrm{P}}}^{(12)}= & \frac{s_{(12)}-m_{1}^{2}+m_{2}^{2}}{2 s_{(12)}} f_{2, \mathrm{a}_{1}\left[\mathrm{f}_{2} \pi\right]_{\mathrm{P}}}^{(12)}+\frac{\text { FIII }}{32 s_{\mathrm{X}}^{2} s_{(12)}^{3}} \\
& \cdot\left[s_{\mathrm{X}}\left(3 s_{(12)}-s_{\mathrm{X}}+m_{3}^{2}\right)-4 s_{\mathrm{X}} s_{(12)}\right. \\
& \left.+\left(m_{3}^{2}-s_{(12)}\right)\left(s_{\mathrm{X}}-m_{3}^{2}+s_{(12)}\right)\right]
\end{aligned}
$$

For completeness, we also give the from factors for orbital angular momenta of three. For the $a_{1}\left[f_{2} \pi\right]_{F}$ wave, we find:

$f_{2, \mathrm{a}_{1}\left[\mathrm{f}_{2} \pi\right]_{\mathrm{F}}}^{(12)}=\frac{3 \mathrm{FI} \cdot \mathrm{FII}}{64 s_{\mathrm{X}}^{2} s_{(12)}}$

and

$$
\begin{aligned}
f_{3, \mathrm{a}_{1}\left[\mathrm{f}_{2} \pi\right]_{\mathrm{F}}}^{(12)}= & \frac{1}{256 s_{\mathrm{X}}^{2} s_{(12)}^{2}}\left[-3 \mathrm{FI} \cdot\left(4 \mathrm{FIII}+\mathrm{FV}^{2}\right.\right. \\
& \left.-2\left(s_{(12)}-m_{1}^{2}+m_{2}^{2}\right) \mathrm{FII}\right) \\
& \left.+2 \mathrm{FIV} \cdot \mathrm{FI}^{2}-15 \mathrm{FII}^{2}\right]
\end{aligned}
$$


The form factors for the $\mathrm{a}_{1}\left[\rho_{3} \pi\right]_{\mathrm{D}}$ are given by:

$$
\begin{aligned}
f_{2, \mathrm{a}_{1}\left[\rho_{3} \pi\right]_{\mathrm{D}}}^{(12)}= & \frac{1}{256 s_{\mathrm{X}}^{2} s_{(12)}^{2}}\left[-3\left(s_{\mathrm{X}}-m_{3}^{2}+s_{(12)}\right)^{2} \mathrm{FIV} \cdot \mathrm{FI}\right. \\
& -15 \mathrm{FII}^{2}+\mathrm{FI} \cdot\left(-20 \mathrm{FIII}-5 \mathrm{FV}^{2}\right)+\mathrm{FIV} \\
& \left.\cdot\left(16 s_{\mathrm{X}} s_{(12)}+\left(s_{\mathrm{X}}-m_{3}^{2}+s_{(12)}\right)^{2}\right)\right]
\end{aligned}
$$

and

$$
\begin{aligned}
f_{3, \mathrm{a}_{1}\left[\rho_{3} \pi\right]_{\mathrm{D}}}^{(12)}= & -\frac{1}{512 s_{\mathrm{X}}^{3} s_{(12)}^{4}}\left[\left(10 \mathrm{FV}^{2}\left(s_{(12)}-m_{1}^{2}+m_{2}^{2}\right)\right.\right. \\
& \left.\cdot s_{\mathrm{X}} s_{(12)}+4 \mathrm{FIIIFV} \cdot\left(s_{\mathrm{X}}-m_{3}^{2}+s_{(12)}\right)\right) \\
& \left(\left(s_{\mathrm{X}}+s_{(12)}-m_{3}^{2}\right)^{2}+2 s_{\mathrm{X}} s_{(12)}\right) \\
& +3 \mathrm{FI} \cdot \mathrm{FIII}\left(s_{(12)}-m_{1}^{2}+m_{2}^{2}\right)\left(s_{\mathrm{X}}-m_{3}^{2}+s_{(12)}\right)^{2} \\
& +\mathrm{FIII} \cdot\left(s_{(12)}-m_{1}^{2}+m_{2}^{2}\right) \\
& \left.\left(\left(s_{\mathrm{X}}+m_{3}^{2}-s_{(12)}\right)^{2}-4 s_{\mathrm{X}} m_{3}^{2}\right)^{2}\right] .
\end{aligned}
$$

Below we give the scalar form factors for arbitrary final-state masses. For the $\pi^{\prime}\left[\mathrm{f}_{0} \pi\right]_{\mathrm{S}}$ wave, we again find $f_{\mathrm{S}, \pi^{\prime}\left[\mathrm{f}_{0} \pi\right]_{\mathrm{S}}}^{(12)}=1$ and for the $\pi^{\prime}[\rho \pi]_{\mathrm{P}}$ we find:

$f_{\mathrm{s}, \pi^{\prime}[\rho \pi]_{\mathrm{P}}}^{(12)}=-2 / 3 f_{2, \mathrm{a}_{1}\left[\mathrm{f}_{2} \pi\right]_{\mathrm{P}}}^{(12)}$.

The scalar form factors for partial waves involving $f_{2}$ and $\rho_{3}$ isobars are given by:

$$
\begin{aligned}
f_{\mathrm{s}, \pi^{\prime}\left[\mathrm{f}_{2} \pi\right]_{\mathrm{D}}}^{(12)}= & \frac{1}{256 s_{\mathrm{X}}^{2} s_{(12)}^{2}}\left[-\left(s_{\mathrm{X}}^{2}-2 s_{\mathrm{X}}\left(m_{3}^{2}-5 s_{(12)}\right)\right.\right. \\
& \left.+\left(m_{3}^{2}-s_{(12)}\right)^{2}-3\left(s_{\mathrm{X}}-m_{3}^{2}+s_{(12)}\right)^{2}\right) \\
& \cdot \mathrm{FIV} \cdot \mathrm{FI}+9\left(s_{\mathrm{X}}-m_{3}^{2}+s_{(12)}\right)^{2} \mathrm{FV}^{2} \\
& \left.+3 \mathrm{FI}\left(4 \mathrm{FIII}+\mathrm{FV}^{2}\right)\right]
\end{aligned}
$$

and

$$
\begin{aligned}
f_{\mathrm{s}, \pi^{\prime}\left[\rho_{3} \pi\right]_{\mathrm{F}}}^{(12)}= & \frac{\mathrm{FII}}{200 s_{\mathrm{X}}^{3} s_{(12)}^{3}}\left[15\left(s_{\mathrm{X}}-m_{3}^{2}+s_{(12)}\right)^{2} \mathrm{FIV} \cdot \mathrm{FI}\right. \\
& -3 \mathrm{FIV} \cdot \mathrm{FI}\left(-3 \mathrm{FI}+20 s_{\mathrm{X}} s_{(12)}\right) \\
& \left.+25 \mathrm{FII}^{2}+15 \mathrm{FI}\left(4 \mathrm{FIII}+\mathrm{FV}^{2}\right)\right] .
\end{aligned}
$$

Since in contrast to axial-vector and pseudo-scalar contributions, vector contributions are suppressed by $G$-parity, we do not give explicit expressions for vector form factors involving higher orbital angular momenta.

\section{References}

1. P.R. Burchat, SLAC-0292 (1986)

2. S. Weinberg, Phys. Rev. 112, 1375-1379 (1958). https://doi.org/ 10.1103/PhysRev.112.1375

3. I.S. Towner, J.C. Hardy, (1995). arXiv:nucl-th/9504015

4. P.A. Zyla et al., Prog. Theor. Exp. Phys. 2020, 083C01 (2020) (Particle Data Group)

5. D.M. Asner et al. (CLEO), Phys. Rev. D 61, 012002 (1999). arXiv:hep-ex/9902022

6. P. Salvini et al. (OBELIX), Eur. Phys. J. C 35, 21-33 (2004). https:// doi.org/10.1140/epjc/s2004-01811-8

7. S.U. Chung, K. Danyo, R.W. Hackenburg, C. Olchanski, J.S. Suh, H.J. Willutzki, S.P. Denisov, V. Dorofeev, V.V. Lipaev, A.V. Popov et al., Phys. Rev. D 65, 072001 (2002). https://doi.org/10.1103/ PhysRevD.65.072001

8. J.H. Kühn, E. Mirkes, Z. Phys. C 56, 661-672 (1992). https://doi. org/10.1007/BF01474741 [erratum: Z. Phys. C 67 (1995), 364]

9. G. Ascoli, D.V. Brockway, H.B. Crawley, B.I. Eisenstein, R.W. Hanft, M.L. Ioffredo, U.E. Kruse, Phys. Rev. Lett. 25, 962 (1970). https://doi.org/10.1103/PhysRevLett.25.962

10. M. Jacob, G.C. Wick, Ann. Phys. 7, 404-428 (1959). https://doi. org/10.1016/0003-4916(59)90051-X

11. H. Albrecht et al. (ARGUS), Z. Phys. C 58, 61-70 (1993). https:// doi.org/10.1007/BF01554080

12. N. Isgur, C. Morningstar, C. Reader, Phys. Rev. D 39, 1357 (1989)

13. M. Mikhasenko et al. (JPAC), Phys. Rev. D 98(9), 096021 (2018). https://doi.org/10.1103/PhysRevD.98.096021. arXiv:1810.00016 [hep-ph]

14. A.D. Martin, T.D. Spearman, Elementary-particle theory (NorthHolland, 1970)

15. A. Jackura et al. (JPAC and COMPASS), Phys. Lett. B 779, 464-472 (2018). https://doi.org/10.1016/j.physletb.2018.01.017. arXiv:1707.02848 [hep-ph]

16. A.V. Anisovich, V.V. Anisovich, V.N. Markov, M.A. Matveev, A.V. Sarantsev, J. Phys. G 28, 15-32 (2002). arXiv:hep-ph/0105330

17. C. Adolph et al. (COMPASS), Phys. Rev. D 95(3), 032004 (2017). https://doi.org/10.1103/PhysRevD.95.032004. arXiv:1509.00992 [hep-ex]

18. M.G. Alexeev et al. (COMPASS), (2021). arXiv:2108.01744 [hepex]

19. D. GomezDumm, A. Pich, J. Portoles, Phys. Rev. D 69, 073002 (2004). https://doi.org/10.1103/PhysRevD.69.073002 arXiv:hep-ph/0312183

20. W. Bernreuther, L. Chen, O. Nachtmann, (2021). arXiv:2101.08071 [hep-ph]

21. M. Feindt, Z. Phys. C 48, 681-688 (1990)

22. S.U. Chung, (2021). https://doi.org/10.5170/CERN-1971-008

23. S.U. Chung, J. Friedrich, Phys. Rev. D 78, 074027 (2008). arXiv:0711.3143 [hep-ph] 